\title{
A Gap Prepulse with a Principal Stimulus Yields a Combined Auditory Late Response
}

\author{
Jae-Hun Lee ${ }^{1}$, Jae Yun Jung ${ }^{1,2}$, and Ilyong Park ${ }^{1,3}$ \\ ${ }^{1}$ Beckman Laser Institute Korea, College of Medicine, Dankook University, Cheonan, Korea \\ ${ }^{2}$ Department of Otolaryngology Head and Neck Surgery, Dankook University Hospital, Cheonan, Korea \\ ${ }^{3}$ Department of Biomedical Engineering, College of Medicine, Dankook University, Cheonan, Korea
}

\author{
Received September 17, 2019 \\ Revised November 13, 2019 \\ Accepted January 23, 2020
}

Background and Objectives: The gap prepulse inhibition of the acoustic startle response has been used to screen tinnitus in an animal model. Here, we examined changes in the auditory late response under various conditions of gap prepulse inhibition. Subjects and Methods: We recruited 19 healthy adults (5 males, 14 females) and their auditory late responses were recorded after various stimuli with or without gap prepulsing. The N1 and P2 responses were selected for analysis. The gap prepulse inhibition was estimated to determine the optimal auditory late response in the gap prepulse paradigm. Results: We found that the gap per se generated a response that was very similar to the response elicited by sound stimuli. This critically affected the gap associated with the maximal inhibition of the stimulus response. Among the various gap-stimulus intervals (GSIs) between the gap and principal stimulus, the GSI of $150 \mathrm{~ms}$ maximally inhibited the response. However, after zero padding was used to minimize artifacts after a P2 response to a gap stimulus, the differences among the GSIs disappeared. Conclusions: Overall, the data suggest that both the prepulse inhibition and the gap per se should be considered when using the gap prepulse paradigm to assess tinnitus in humans.

J Audiol Otol 2020;24(3):149-156

\section{Introduction}

An auditory evoked response (AER) is an electrical potential evoked by an external auditory stimulus. The AER is thought to provide useful neurophysiological information because of its sensitive temporal resolution. Among the various AER components, the N1 and P2 peaks of the auditory late response (ALR) represent auditory perception and provide information about the psychological state of an individual [1]. Experiments using a model of neurophysiological tinnitus indicated that these components affect tinnitus [1,2]. The N1 and P2 components differ significantly between tinnitus patients and healthy subjects [2].

This is an Open Access article distributed under the terms of the Creative Commons Attribution Non-Commercial License (https://creativecommons.org/licenses/by-nc/4.0/) which permits unrestricted non-commercial use, distribution, and reproduction in any medium, provided the original work is properly cited.
Prepulse inhibition (PPI) is a general neurological phenomenon wherein the magnitude of a response to a principal stimulus is reduced when a certain stimulus precedes it by $30-$ $500 \mathrm{~ms}$ [3]. PPI is indicative of sensorimotor gating and can be affected by various factors such as drugs, attention, and psychological issues $[4,5]$. Recent studies have demonstrated that prepulsing reduced the magnitudes of the cortical auditory evoked potentials (AEPs; P50, N1, P2, and P300) in healthy humans [5].

Advances in the methods of tinnitus assessment in animals have facilitated significant developments in tinnitus research. Turner, et al. [6] used a novel method termed gap PPI (gPPI) of the acoustic startle reflex to screen for tinnitus in an animal model. This new paradigm is based on the concept that a gap in the auditory stimulus serves as a prepulse and affects the acoustic startle response. By providing a gap before the main stimulus, in the presence of background noise similar to tinni- 
tus, the startle response of an animal was reduced due to PPI. However, such inhibition was not evident in animals with tinnitus. Several studies have used this model to measure behavioral changes in various animals with noise-induced tinnitus $[7,8]$.

Previous studies have investigated the relationship between the human startle reflex and PPI in the presence of various levels of background noise; a prepulse sound inhibited the startle response in humans $[9,10]$. In humans, an eye-blink startle response is typically measured using electromyography of the orbicularis oculi muscle [3]. Some studies have reported an inhibited response after a small pulse sound, especially in patients with insomnia [11] and traumatic stress disorder [12]. We speculate that the prepulse paradigm can also be used for evaluating humans; the startle response is replaced by the ALR when tinnitus is present.

$\mathrm{Ku}$, et al. [13] used the gPPI paradigm with humans to evaluate if it could be used for the objective assessment of tinnitus. It was found that the auditory responses of the N1P2 complex were stable. The N1P2 ratios under gap and no-gap conditions differed marginally, depending on the gap duration, gap-stimulus interval (GSI), level of background noise, and response attained at the maximum noise level with the shortest gap duration $(20 \mathrm{~ms})$ and the lowest level of background noise. However, as the recording of AEPs with short repetition rates is uncommon because of overlapping neural responses [14], an ALR may include a response due to a preceding gap if there is only a short interval prior to the main stimulus. This possibility should be considered if a gap serves as an "auditory stimulus" that can itself evoke an auditory response.
We explored the possibility that a "gap" can serve as a stimulus for generating an auditory response at the cortical level. We also examined the feasibility of the gPPI paradigm for ALR measurement and investigated the differences in gPPI values derived using various GSIs between a gap prepulse and the principal pulse. Additionally, we investigated the effects of gap-only stimuli in the gap prepulse paradigm by correcting for the responses evoked by the gap prepulse stimuli. Our results demonstrate how the positioning of the gap prepulse affects the gPPI.

\section{Subjects and Methods}

\section{Subjects}

We enrolled 19 adults ( 5 males, 14 females) with a mean age of 28.68 years (range $21-50$ years). None of the participants had any history of tinnitus or neurological disease. Their hearing was normal, as shown by pure tone audiometry at 500, 1,000, 2,000, and 4,000 Hz; all hearing thresholds were below $25 \mathrm{~dB}$ HL (hearing level) at all tested frequencies. This study was approved by the Institutional Board of Dankook University Hospital (DKUH 2013-08-009).

\section{ALR measurement}

The system used to measure ALR is described in Fig. 1. ALR potentials were measured using the wireless electroencephalogram (EEG) neuroheadset device (Epoc, EMOTIV Inc., San Francisco, CA, USA) and the computer-based custom data acquisition (DAQ) (NI PXI-6251, National Instruments, Austin, TX, USA) system (ALR system algorithm)

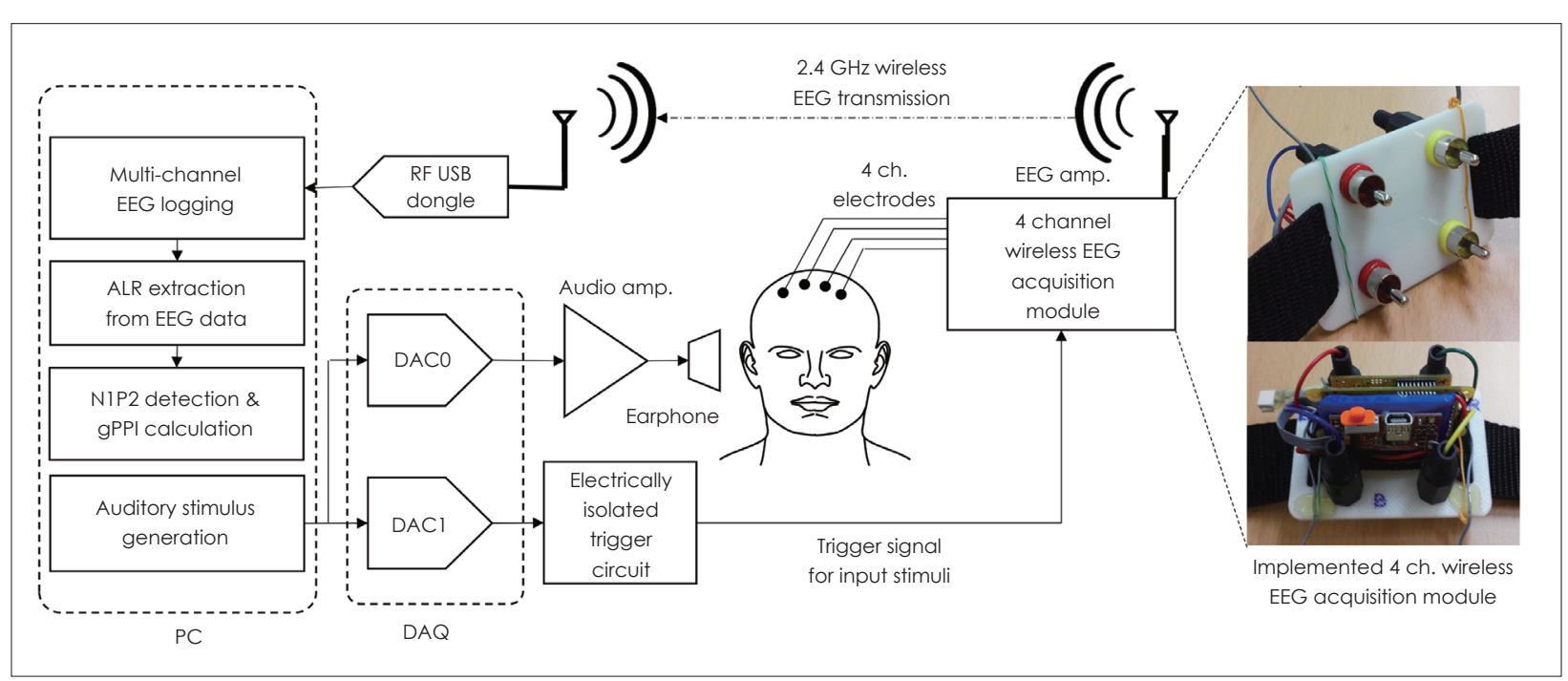

Fig. 1. Auditory late response (ALR) measurement. Stimuli were generated by the computer and presented via earphones. Responses were recorded using a four-channel wireless electroencephalogram (EEG) acquisition module and sent to the computer for analysis. ch.: channel, RF: radio frequency, DAC: digital-to-analog converter, gPPI: gap PPI, PC: personal computer, DAQ: data acquisition. 
(Fig. 1). The system featured the DAQ software, EEG amplification and logging software, an EEG trigger, and the auditory response signal collection software.

\section{Stimulus and response processing}

The stimuli used are shown in Fig. 2. All stimuli included a continuous background noise with duration of 1,000 ms. The principal pulse (white noise) was $80 \mathrm{~dB}$ SPL (sound pressure level) and $20 \mathrm{~ms}$ in duration, delivered within a $500 \mathrm{~ms}$ stimulus train. To measure gap-only responses, stimuli of a $50 \mathrm{~ms}$ gap presented at the $400 \mathrm{~ms}$ time point within the background noise ( $8 \mathrm{kHz}, 1 / 3$ octave band) of $60 \mathrm{~dB}$ SPL was used (Fig. 2). Except when gap-only stimuli were delivered, both the gap and principal stimuli within the background noise were considered a single stimulus (Fig. 2). Gaps were placed at three different time points in the stimulus train: GSIs of 50, 150, and $250 \mathrm{~ms}$. The stimuli were randomly presented at inter-stimulus intervals (ISIs) of $1-3 \mathrm{~s}$ to avoid adaptation and temporal prediction of the acoustic stimuli, using the ER-A3 (Etymotic Research, Elk Grove Village, IL, USA) insert earphone.

\section{ALR measurements and response processing}

All ALRs were measured in a double-walled soundproofed room. Participants were seated in comfortable chairs, and an inspector ensured that they did not sleep or engage in habitual movements. The common mode sense electrode was placed on the ipsilateral ear (reference) and the driven light leg electrode was placed on the contralateral earlobe (ground). Headgear with four channel electrodes (AF3, AF4, F3, and F4) was placed, and measurements commenced when the impedances of all electrodes were $\leq 5 \mathrm{k} \Omega$. Stimuli were delivered (via the earphone) to the left ear, and the contralateral ear was blocked with a sponge earplug (KE-1100, Moden zone, Seoul, Korea). For each subject, three $5 \mathrm{~min}$ measurements at a $128 \mathrm{~Hz}$ sampling rate were made. The responses were processed using a software, and the responses to no-gap and with-gap stimuli were displayed as bold and dotted lines, respectively (Fig. 3).

EEG signals transferred to the computer were processed using specialized software. Both baseline drift and high-frequency noise were removed using a $1-30 \mathrm{~Hz}$ band-pass filter. The responses with or without gap prepulse were averaged. With- and without-gap responses are presented together; the ranges of interest including N1 (first negative peak) and P2 (second positive peak) are highlighted using yellow bars. The amplitudes of with- and without-gap responses were automatically calculated from the N1P2 amplitude, within the region

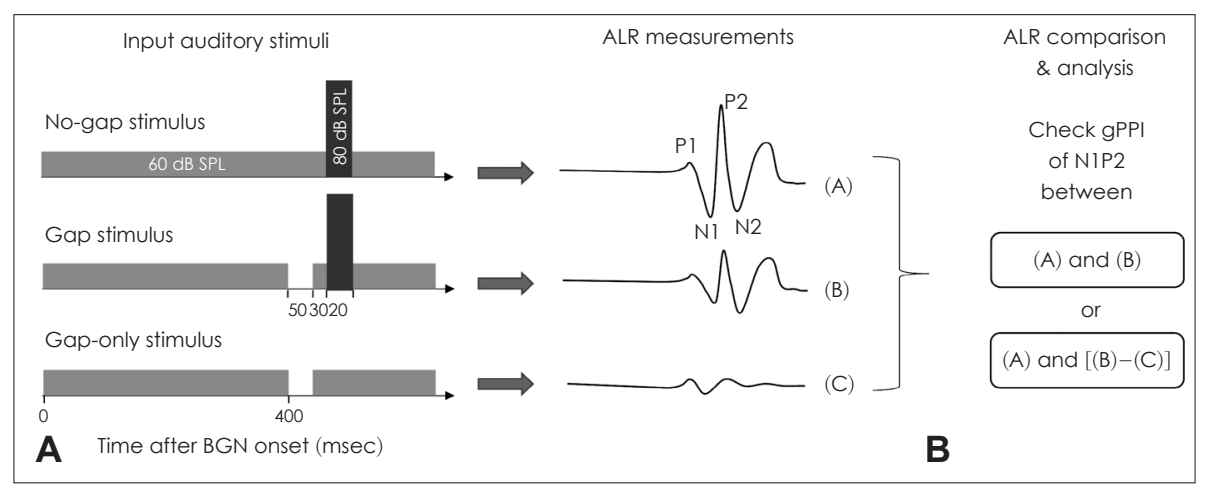

Fig. 2. The stimuli and auditory late response (ALR). A: Various stimuli including background noise with a principal stimulus, gap-ped background with a prin-cipal stimulus, and gapped back-ground alone. The responses to all stimuli were recorded (white numbers represent intensity of stimulus and black numbers represent latency). B: Acquired responses calculated as gap PPI (gPPI) values. "(A)" indicates "the response from No-gap stimulus". "(B)" indicates "the response from gap stimulus". "(C)" indicates "the response from gap-only stimulus". BGN: background noise.

Fig. 3. Auditory late responses (ALRs) with- and without-gap prepulsing. The bold line indicates the response to the principal stimulus ( $80 \mathrm{~dB}$ SPL) and the dotted line indicates the response to a gap followed by the principal stimulus. The background noise was narrow-band noise of $8 \mathrm{kHz}$ with a bandwidth of $1 \mathrm{kHz}$ (60 dB SPL). BGN: background noise.

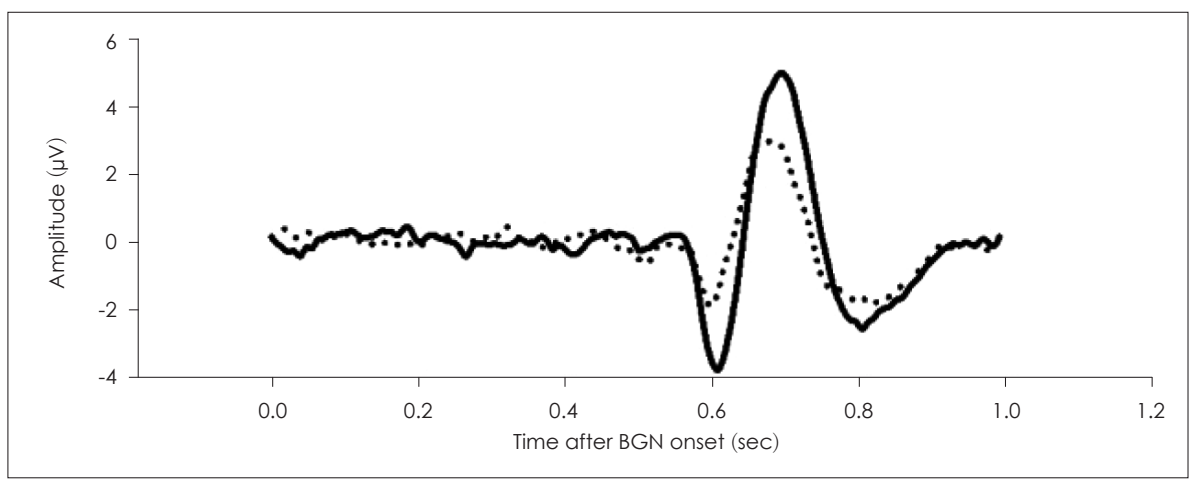


of interest.

\section{Data analysis}

Epoch durations of 1,000 ms (which are sufficient to include all responses to the longest GSI) were analyzed. Four peaks developing within 0.4 to $0.8 \mathrm{~ms}$ were measured: N1, $\mathrm{P} 1, \mathrm{~N} 2$, and $\mathrm{P} 2$ peaks. Of these, the N1 and P2 peaks after both no-gap and gap stimuli were automatically identified, and the gPPI was defined using the following equation:

$$
\mathrm{gPPI}=\frac{\mathrm{N} 1 \mathrm{P} 2_{\text {no gap }}-\mathrm{N} 1 \mathrm{P} 2_{\text {gap }}}{\mathrm{N} 1 \mathrm{P} 2_{\text {no gap }}}
$$

Zero padding was applied to the corrected data. The responses after the zero-crossing points of P2N2 waves after gap-only stimuli were set to zero. Thus, the ALR gap-only response was subtracted from the response after both a gap and a principal pulse. Mean and standard deviation (mean \pm SD) of all peak amplitudes were compared.

\section{Statistical analysis}

All data were analyzed using the Statistical Package for the Social Sciences ver. 19 (IBM, Armonk, NY, USA). Analysis of variance (ANOVA) with Bonferroni post-hoc and paired ttest were used to determine the effect of GSI on the gPPI.

\section{Results}

\section{Effects of gap prepulsing on N1P2 amplitudes at different GSIs}

All responses were averaged, yielding the grand-averaged data. The grand-averaged ALR after an $80 \mathrm{~dB}$ SPL $(8 \mathrm{kHz})$

Table 1. N1P2 inter-peak amplitudes (mean \pm standard deviation) with- and without-gap prepulsing at different GSIs

\begin{tabular}{cccc}
\hline \multicolumn{1}{c}{ GSI (msec) } & 50 & 15 & 250 \\
\hline No-gap response $(\mu \mathrm{V})$ & $9.77 \pm 3.56$ & $9.71 \pm 3.16$ & $10.6 \pm 4.88$ \\
Gap response $(\mu \mathrm{V})$ & $6.24 \pm 2.42$ & $8.17 \pm 3.00$ & $8.39 \pm 2.61$ \\
\hline
\end{tabular}

GSI: gap-stimulus interval stimulus is shown in Fig. 3. The N1P2 amplitude was $8.66 \mu \mathrm{V}$, and the N1 and P2 latencies were 0.6 and $0.69 \mathrm{~ms}$, respectively. The N1P2 amplitude decreased when a silent gap (50 ms) was inserted prior to the principal stimulus (Fig. 3). In this case, the N1P2 amplitude was $4.96 \mu \mathrm{V}$. The N1P2 amplitudes (mean \pm SD) at different GSIs are listed in Table 1. A GSI of 50 $\mathrm{ms}$ resulted in the greatest decrease in the N1P2 amplitude.

We calculated gPPI values to explore ALR changes at various GSIs. The gPPI values at GSIs of 50, 150, and $250 \mathrm{~ms}$ are shown in Fig. 4. The gPPI was maximal at the GSI of 50 $\mathrm{ms}$ and decreased as the GSI increased. We used the paired $\mathrm{t}$ test to explore how the GSI affected the gPPI. Significant differences between the gPPI values at GSIs of 50 and 150 $\mathrm{ms}(p=0.012)$, and at GSIs of 50 and $250 \mathrm{~ms}(p=0.001)$, were apparent, but not between the gPPI values at GSIs of 150 and $250 \mathrm{~ms}(p=0.435)$.

Effect of the gap prepulse on N1P2 amplitude (after subtracting the gap-evoked auditory response)

The decrease in the N1P2 amplitude when a gap prepulse

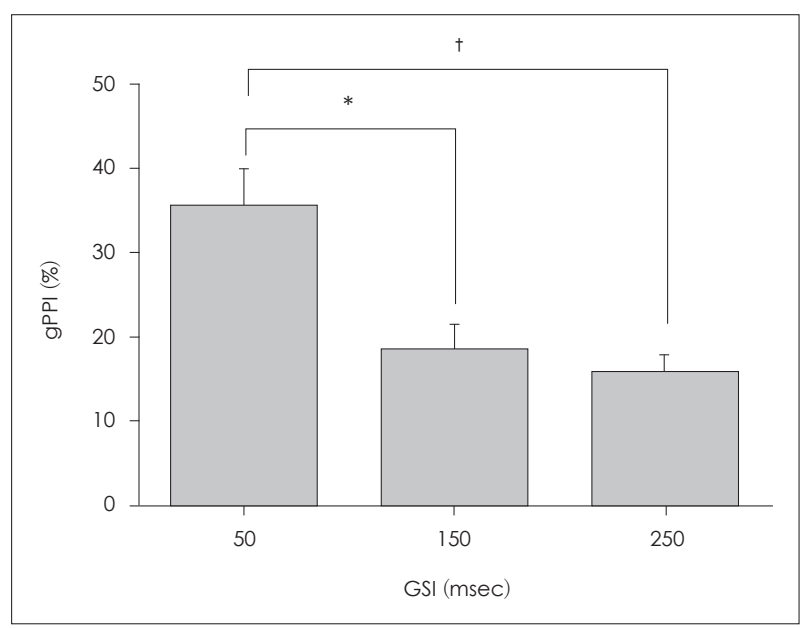

Fig. 4. Gap PPI (gPPI) values (mean \pm standard error of the mean) elicited by different gap-stimulus intervals (GSIs). There was a statistically significant association between the GSI of $50 \mathrm{~ms}$ and the highest gPPI, as compared with the GSIs of $150 \mathrm{~ms}\left({ }^{*} p<0.05\right)$ and $250 \mathrm{~ms}\left({ }^{\dagger} p<0.01\right)$.

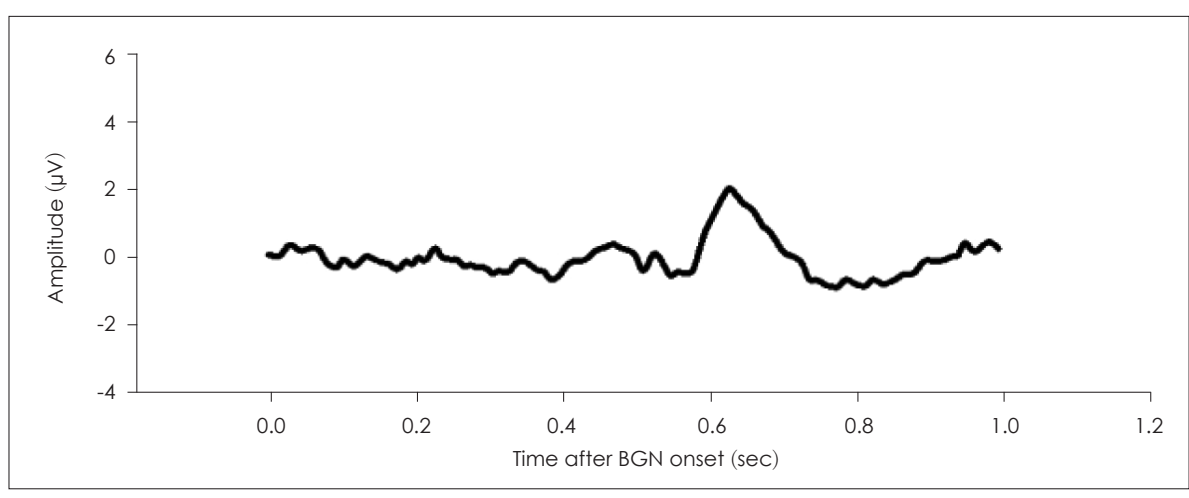

Fig. 5. Auditory late responses elicited by only a gap. The background noise was narrow-band noise of 8 $\mathrm{kHz}$ with a bandwidth of $1 \mathrm{kHz}(60 \mathrm{~dB}$ $\mathrm{SPL})$. Gaps within the background noise evoked auditory responses similar to those evoked by sound stimuli. BGN: background noise. 
was applied prior to the principal stimulus is assumed to reflect the PPI of the N1P2 response. Moreover, the gap prepulse-induced auditory response may generate interference. Previously, several studies have reported that the AEPs evoked within $300 \mathrm{~ms}$ of multiple stimuli changed if they were overlaid with auditory responses $[15,16]$. Thus, we first measured the ALR under gap-only conditions (within background noise), and acquired evoked responses that were morphologically similar to those evoked by the principal sound stimulus (Fig. 5). All peak latencies were also similar $(\mathrm{N} 1=0.56, \mathrm{P} 2=0.64)$. Thus, the decrease in the N1P2 amplitude of the gap response may reflect the simple mathematical summation of the two waveforms (the gap-only and no-gap responses). To explore this possibility, we corrected each ALR response by subtracting the auditory response elicited by the gap-only stimulus, and then averaged the responses to derive a corrected grand-average gPPI. The N1P2 amplitudes after correction of the data obtained at various GSIs are listed in Table 2.

The gPPI values after correction are shown in Fig. 6. The mean \pm SD gPPI values were $18.59 \pm 27.47 \%, 2.26 \pm 24.62 \%$, and $17.93 \pm 12.7 \%$ at GSIs of 50,150 , and $250 \mathrm{~ms}$, respectively. The value was highest at the GSI of $50 \mathrm{~ms}$, decreased markedly at $150 \mathrm{~ms}$, and increased again at $250 \mathrm{~ms}$. We used the paired t-test to examine the effect of the GSI on the corrected gPPI values. The gPPI values at GSIs of 150 and $250 \mathrm{~ms}$ differed sig-

Table 2. Corrected N1P2 inter-peak amplitudes (mean \pm standard deviation) and gPPI values with- and without-gap prepulsing at different GSIs

\begin{tabular}{lccc}
\hline \multicolumn{1}{c}{ GSI (msec) } & 50 & 150 & 250 \\
\hline No-gap response $(\mu \mathrm{V})$ & $9.77 \pm 3.56$ & $9.71 \pm 3.16$ & $10.6 \pm 4.88$ \\
Gap response $(\mu \mathrm{V})$ & $7.53 \pm 2.83$ & $9.23 \pm 3.11$ & $7.55 \pm 2.84$ \\
\hline
\end{tabular}

GSI: gap-stimulus interval

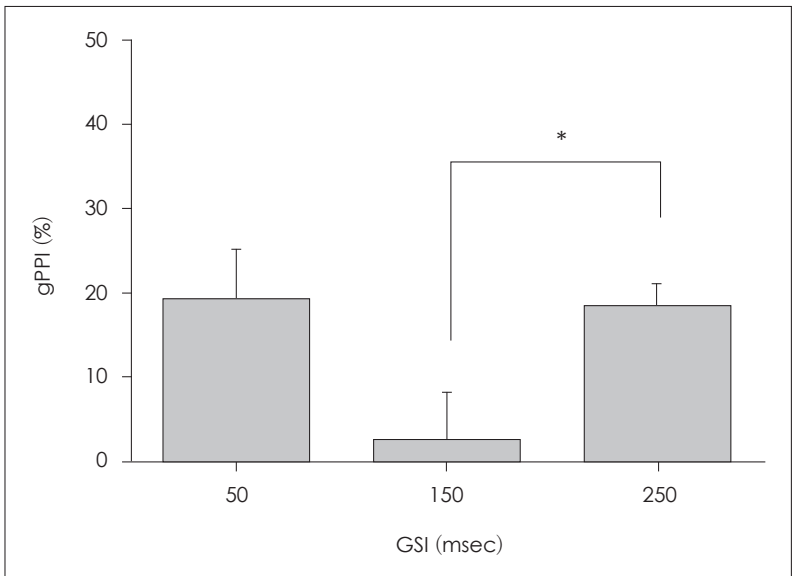

Fig. 6. Corrected gap PPI (gPPI) values (mean \pm standard error of the mean). After correction (subtraction of the gap-only stimulus), the gap-stimulus interval (GSI) of $150 \mathrm{~ms}$, but not $250 \mathrm{~ms}$, was associated with a near-zero gPPI ( $\left.{ }^{*} p<0.05\right)$. nificantly ( $p=0.011$ ), but those at GSIs of 50 and $150 \mathrm{~ms}(p=$ $0.058)$ and 50 and $250 \mathrm{~ms}(p=0.922)$ did not.

\section{The gPPI values after zero padding}

ALRs can be classified as exogenous and endogenous responses, depending on latency [17]. An exogenous response is highly dependent on the physical properties of the stimulus, whereas an endogenous response varies with the characteristics of the stimulus and psychological status of the subject [17]. As PPI was apparent even after a very brief GSI, we considered that such inhibition was more indicative of an exogenous rather than an endogenous response. Thus, we zero padded the gap-only response to minimize any effect of an endogenous response. All responses after zero-padding correction were averaged to obtain corrected grand-averaged gPPI values. The values at GSIs of 50, 150, and $250 \mathrm{~ms}$ are shown in Fig. 7. The highest value occurred at the GSI of $50 \mathrm{~ms}$, and the lowest at the GSI of $250 \mathrm{~ms}$.

All gPPI values associated with gap-only stimuli were subjected to zero-padding correction (Table 3 ). The mean \pm SD gPPI values were $18.31 \pm 22.17 \%, 14.49 \pm 17.16 \%$, and $13.07 \pm$ $11.73 \%$ at GSIs of 50, 150, and $250 \mathrm{~ms}$, respectively (Fig. 8). The highest gPPI value occurred at the GSI of $50 \mathrm{~ms}$ and then decreased as the GSI increased. We used the paired t-test to analyze the effect of the GSI; no difference was apparent among the GSIs of 50,150 , and $250 \mathrm{~ms}$.

\section{Discussion}

We sought to identify ALR changes after gPPI in humans with normal hearing. First, we investigated gPPI under conditions very similar to those used in animal studies, and found

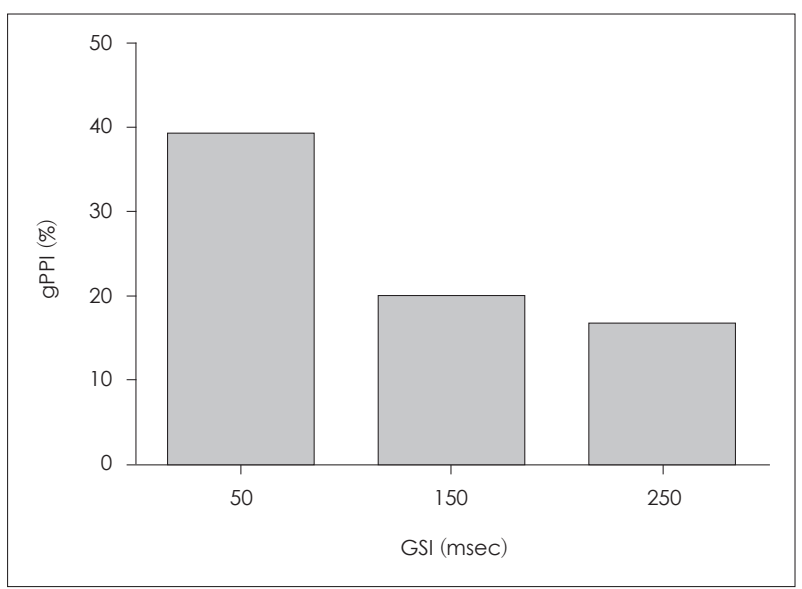

Fig. 7. Corrected grand-averaged gap PPI (gPPI) values after zero padding using different gap-stimulus intervals (GSIs). After zero padding, among the three GSIs evaluated, the GSI of $50 \mathrm{~ms}$ was associated with the highest gPPI. Notably, GSIs of 250 and $500 \mathrm{~ms}$ resulted in similar degrees of inhibition. 
Table 3. Corrected N1P2 inter-peak amplitudes including those obtained after zero padding (mean \pm standard deviation) with- and without-gap prepulsing at different GSIs

\begin{tabular}{lccc}
\hline \multicolumn{1}{c}{ GSI (msec) } & 50 & 150 & 250 \\
\hline No-gap response $(\mu \mathrm{V})$ & $9.77 \pm 3.56$ & $9.71 \pm 3.16$ & $10.6 \pm 4.88$ \\
Gap response $(\mu \mathrm{V})$ & $7.71 \pm 2.78$ & $8.28 \pm 2.93$ & $8.39 \pm 2.61$ \\
\hline
\end{tabular}

GSI: gap-stimulus interval

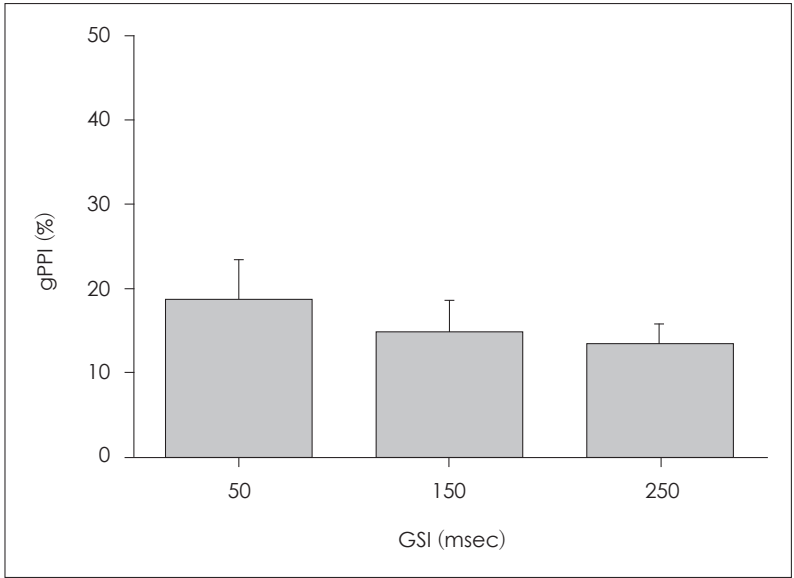

Fig. 8. The gap PPI (gPPI) values (mean \pm standard error of the mean) after zero-padding correction. After correction, the gPPI did not vary according to the gap-stimulus interval (GSI).

that the ALR was inhibited by a gap prepulse. This was in line with the assumption that an AER can be generated using the gap prepulse paradigm [18]. Recently, various gap parameters have been shown to inhibit the effects of a gap prepulse on the ALR [19] and the eye-blink reflex in healthy adults [20]. Our results are similar to those of $\mathrm{Ku}$, et al. [13]. Furthermore, although direct comparisons of our data with those of animal studies would be inappropriate, we measured human responses derived using the gap prepulse paradigm; the results were similar to those of animal studies. Therefore, we support the use of the gap prepulse paradigm for tinnitus assessment in humans.

The ALR responses obtained using various GSIs showed that gap placement $50 \mathrm{~ms}$ prior to the principal stimulus resulted in the greatest inhibition of the response. Previous work on gPPI focused on gap duration; a longer gap prepulse was associated with greater inhibition, both for a simple gap prepulse and a gap following a prepulse when background noise was lacking between the gap and principal pulse [21]. Although a recent study on gPPI in healthy adult humans reported that the GSI did not affect the response [13], all GSIs were $<100$ ms. It is possible that other factors (such as gap offset or onset and background noise) affect the ALR response. Thus, the study does not confirm that the GSI between the gap and the principal stimulus is irrelevant in the context of the gap prepulse paradigm. We found that responses varied signifi- cantly with the GSI, even after the gap-only stimulus was subtracted. Our data and previous results suggest that further studies using GSIs of various durations $<100 \mathrm{~ms}$, with gap response subtraction, are required to confirm whether the GSI influences the gap prepulse paradigm in humans.

We found that the GSI of $250 \mathrm{~ms}$ also inhibited the response to the principal stimulus, albeit not as markedly as the GSIs of 50 and $150 \mathrm{~ms}$. However, the GSI of $250 \mathrm{~ms}$ is clinically relevant, and temporal summation is impossible. Blumenthal [9] reported that the extent of the eye-blink inhibition by a prepulse depended on the prepulse duration and the interval between the prepulse offset and the onset of the principal pulse (the GSI in our present study). However, all GSIs applied were between 100 and $144 \mathrm{~ms}$. Furthermore, whether the GSI of $50 \mathrm{~ms}$ was more inhibitory than GSIs of 150 or $250 \mathrm{~ms}$ was not investigated. Therefore, the ALR response under gap prepulse conditions is influenced not only by gap duration but also by a GSI $>150 \mathrm{~ms}$.

To identify the pure inhibitory effects of all GSIs, we subtracted the responses evoked by gap prepulsing; such prepulses were not inhibitory at the GSI of $150 \mathrm{~ms}$. This is an interesting finding as it shows that the effect of the gap prepulse on the ALR depends on the GSI, and at some GSIs, the effect is saturated. We considered many other factors that might explain this result. As the AEP is very sensitive to measurement conditions and long measurement times, extreme caution is required during experiments for estimating such potentials. Grand-averaged data indicate gPPI trends. We sought to average all data obtained using the same GSIs; these are the most generalizable results of our study. Although statistical analysis was not possible, the most prominent inhibition was associated with the GSI of $50 \mathrm{~ms}$, and the ALR response after a gap prepulse was more inhibited at shorter GSIs. Previous studies have proposed the use of the gap prepulse inhibition paradigm for tinnitus assessment in clinics [13,22]. We also concluded that it could be a useful assessment tool for patients with tinnitus. However, according to our results, the gap prepulse itself and application of zero padding affect the result of the auditory brainstem response (ABR) after a gap prepulse stimulus, and these effects should be considered in this paradigm.

The N1P2 peak has been used to evaluate cochlear implants [23], explore the effects of drugs on auditory cognition [24], and functionally assess patients with schizophrenia [25]. Ku, et al. [13] reported that the N1P2 amplitude peak was the most prominent of the various ALR peaks (N1, P2, $\mathrm{N} 2$, N1P2, and P2N2) in the gap prepulse paradigm. These studies suggest that our evaluation of the N1P2 peak of the gPPI ratio is appropriate when applying the paradigm. Sensory gating (inhibition of the AER by a prepulse) has also 
been evaluated [26-28]. Several works have explored the relationship between the eye-blink response and the AER (especially N1P2) in the context of the prepulse paradigm, and suggested that different mechanisms are in play $[27,28]$. As the eye-blink is a behavioral response that is easily influenced by a subject's intention, assessment of an AER, especially an N1P2 response, is appropriate for tinnitus evaluation. However, the utility of the paradigm for children should be carefully considered, as ALR responses do not fully represent auditory sensitivity until late adolescence.

Multiple or paired stimuli delivered at various GSIs have been used to evaluate the auditory processing capacity [29]. However, when evaluating cortical responses, rapid re-presentation rates trigger response overlaps, making it difficult to interpret the results [14]. We found that a gap per se in the background noise evoked auditory responses. The response patterns associated with gaps in the background noise were very similar to the responses to sound stimuli. The relatively large baseline fluctuation noted in a previous study wherein gap prepulse stimuli was applied [19] may be attributed to overlaps. A recent study showed that use of the gPPI paradigm was associated with similar auditory responses in both tinnitus patients and normal subjects, perhaps because of a similar issue with overlap [22]. We used zero padding to eliminate any possibility of remnant wave fluctuation even after allowing sufficient time for a cortical response. After zero-padding correction, the gPPI did not vary according to the GSI. This challenges the conclusion of a previous study that the AEP varies with the time between the gap and principal stimulus in the gap prepulse paradigm. In conclusion, both the effect of a gap per se and that of the PPI should be considered when using the gap prepulse paradigm to evaluate humans.

\section{Acknowledgments}

This study was supported by the Ministry of Science, Information and Communications technology (ICT) and Future Planning grant funded by the Korean Government (NRF- 2017R1D1A1B03033219).

\section{Conflicts of interest}

The authors have no financial conflicts of interest.

\section{Author Contributions}

Conceptualization: Ilyong Park and Jae Yun Jung. Data curation: JaeHun Lee and Ilyong Park. Formal analysis: Jae-Hun Lee and Jae Yun Jung. Funding acquisition: Jae Yun Jung. Investigation: Jae Yun Jung. Methodology: Jae Yun Jung and Ilyong Park. Project administration: Jae Yun Jung and Ilyong Park. Resources: Ilyong Park. Software: Ilyong Prak. Supervision: Jae Yung Jung and Ilyong Park. Validation: Jae Yun Jung. Writing — original draft: Jae-Hun Lee. Writing — review \& editing: All authors.

\section{ORCID iDs}

Jae-Hun Lee https://orcid.org/0000-0003-2317-9958
Jae Yun Jung

https://orcid.org/0000-0002-1870-0748

Ilyong Park

https://orcid.org/0000-0003-1613-4209

\section{REFERENCES}

1) Norena A, Cransac H, Chéry-Croze S. Towards an objectification by classification of tinnitus. Clin Neurophysiol 1999;110:666-75.

2) Lee CY, Jaw FS, Pan SL, Lin MY, Young YH. Auditory cortical evoked potentials in tinnitus patients with normal audiological presentation. J Formos Med Assoc 2007;106:979-85.

3) Graham FK. Presidential Address, 1974. The more or less startling effects of weak prestimulation. Psychophysiology 1975;12:238-48.

4) Swerdlow NR, Braff DL, Geyer MA. Animal models of deficient sensorimotor gating: what we know, what we think we know, and what we hope to know soon. Behav Pharmacol 2000;11:185-204.

5) Kedzior KK, Martin-Iverson MT. Chronic cannabis use is associated with attention-modulated reduction in prepulse inhibition of the startle reflex in healthy humans. J Psychopharmacol 2006;20:471-84.

6) Turner JG, Brozoski TJ, Bauer CA, Parrish JL, Myers K, Hughes LF, et al. Gap detection deficits in rats with tinnitus: a potential novel screening tool. Behav Neurosci 2006;120:188-95.

7) Chen G, Lee C, Sandridge SA, Butler HM, Manzoor NF, Kaltenbach JA. Behavioral evidence for possible simultaneous induction of hyperacusis and tinnitus following intense sound exposure. J Assoc Res Otolaryngol 2013;14:413-24.

8) Lobarinas E, Hayes SH, Allman BL. The gap-startle paradigm for tinnitus screening in animal models: limitations and optimization. Hear Res 2013;295:150-60.

9) Blumenthal TD. Prepulse inhibition of the startle eyeblink as an indicator of temporal summation. Percept Psychophys 1995;57:487-94.

10) Braff DL, Geyer MA, Swerdlow NR. Human studies of prepulse inhibition of startle: normal subjects, patient groups, and pharmacological studies. Psychopharmacology (Berl) 2001;156:234-58.

11) Hairston IS, Talbot LS, Eidelman P, Gruber J, Harvey AG. Sensory gating in primary insomnia. Eur J Neurosci 2010;31:2112-21.

12) Grillon C, Morgan CA 3rd, Davis M, Southwick SM. Effects of experimental context and explicit threat cues on acoustic startle in Vietnam veterans with posttraumatic stress disorder. Biol Psychiatry 1998;44:1027-36.

13) Ku Y, Ahn JW, Kwon C, Suh MW, Lee JH, Oh SH, et al. Gap prepulse inhibition of the auditory late response in healthy subjects. Psychophysiology 2015;52:1511-9.

14) Ozdamar O, Bohórquez J. Signal-to-noise ratio and frequency analysis of continuous loop averaging deconvolution (CLAD) of overlapping evoked potentials. J Acoust Soc Am 2006;119:429-38.

15) Burkard RF, Eggermont JJ, Don M. Stimuli for auditory evoked potential assessment. Auditory evoked potentials: basic principles and clinical application. 1st ed. Baltimore, USA: Lippincott Williams \& Wilkins;2007. p.42-73.

16) Sussman E, Steinschneider M, Gumenyuk V, Grushko J, Lawson K. The maturation of human evoked brain potentials to sounds presented at different stimulus rates. Hear Res 2008;236:61-79.

17) Squires KC, Hecox KE. Electrophysiological evaluation of higher level auditory processing. Seminars in Hearing. New York, USA: Thieme Medical Publishers, Inc.;1983. p.415-32.

18) Palmer SB, Musiek FE. N1-p2 recordings to gaps in broadband noise. J Am Acad Audiol 2013;24:37-45.

19) Kujawa SG, Liberman MC. Synaptopathy in the noise-exposed and aging cochlea: primary neural degeneration in acquired sensorineural hearing loss. Hear Res 2015;330:191-9.

20) Graham SJ, Scaife JC, Balboa Verduzco AM, Langley RW, Bradshaw $\mathrm{CM}$, Szabadi E. Effects of quetiapine and haloperidol on prepulse inhibition of the acoustic startle (eyeblink) response and the N1/P2 auditory evoked response in man. J Psychopharmacol 2004;18:173-80.

21) Fournier P, Hébert $S$. The gap-startle paradigm to assess auditory 
temporal processing: bridging animal and human research. Psychophysiology 2016;53:759-66.

22) Ku Y, Ahn JW, Kwon C, Kim DY, Suh MW, Park MK, et al. The gapprepulse inhibition deficit of the cortical N1-P2 complex in patients with tinnitus: the effect of gap duration. Hear Res 2017;348:120-8.

23) Firszt JB, Chambers RD, Kraus And N, Reeder RM. Neurophysiology of cochlear implant users I: effects of stimulus current level and electrode site on the electrical ABR, MLR, and N1-P2 response. Ear Hear 2002;23:502-15.

24) Graham SJ, Langley RW, Bradshaw CM, Szabadi E. Effects of haloperidol and clozapine on prepulse inhibition of the acoustic startle response and the N1/P2 auditory evoked potential in man. J Psychopharmacol 2001;15:243-50.

25) Shahriari Y, Krusienski D, Dadi YS, Seo M, Shin HS, Choi JH. Impaired auditory evoked potentials and oscillations in frontal and auditory cortex of a schizophrenia mouse model. World J Biol Psychi- atry 2016;17:439-48.

26) Inui $K$, Tsuruhara A, Kodaira M, Motomura E, Tanii H, Nishihara $\mathrm{M}$, et al. Prepulse inhibition of auditory change-related cortical responses. BMC Neurosci 2012;13:135.

27) Phillips MA, Langley RW, Bradshaw CM, Szabadi E. The effects of some antidepressant drugs on prepulse inhibition of the acoustic startle (eyeblink) response and the N1/P2 auditory evoked response in man. $\mathrm{J}$ Psychopharmacol 2000;14:40-5.

28) Phillips MA, Oxtoby EK, Langley RW, Bradshaw CM, Szabadi E. Effects of acute tryptophan depletion on prepulse inhibition of the acoustic startle (eyeblink) response and the N1/P2 auditory evoked response in man. J Psychopharmacol 2000;14:258-65.

29) Shi L, Chang Y, Li X, Aiken SJ, Liu L, Wang J. Coding deficits in noise-induced hidden hearing loss may stem from incomplete repair of ribbon synapses in the cochlea. Front Neurosci 2016;10:231. 